\title{
Serum Sickness in Patients Receiving Equine Rabies Immunoglobulin
}

\author{
Sabita Maharana', Tapas Ranjan Behera' ${ }^{2}$ Nupur Pattanaik ${ }^{3}$ \\ ${ }^{1}$ Post Graduate Trainee, ${ }^{2}$ Assistant Professor, ${ }^{3}$ Senior Resident, Department of Community Medicine, \\ SCB Medical College, Cuttack, Odisha, India. \\ DOI: https://doi.org/10.24321/0019.5138.201811
}

\begin{abstract}
Background: Equine Rabies Immunoglobulin (ERIG) is being used for treatment of category-III animal bite cases. ERIG is economical as compared to Human Rabies Immunoglobulin (HRIG) and hence more affordable to patients in developing countries, like India. Despite high degree of purification, there are reported cases of serum sickness like reactions, anaphylaxis, and other allergic reactions following ERIG administration. Serum sickness is a type III or immune-complex-mediated hypersensitivity reaction that results from the injection of heterologous or foreign protein or serum. Because of fear of side effects (including serumsickness-like reaction) following ERIG, the use of ERIG is minimal among doctors.

Objective: (1) To assess the incidence of serum sickness following the administration of ERIG. (2) To see the clinical features of serum sickness.

Methods: This is a hospital-based cross-sectional study carried out in the ARV OPD of SCB Medical College, Cuttack, Odisha, India. All patients who received ERIG between September 2016 and December 2016 were included in the study. Side effects were monitored during the subsequent follow-up visits.

Results: Out of 1548 category-III animal bite cases who received ERIG, 3.1\% developed serum-sickness-like reactions, which manifested as rashes in $97.82 \%$. Most of them had symptoms after seventh day following ERIG administration. Symptomatic relief with oral antihistamines like Cetirizine, Levocetirizine in $60 \%$ of cases and Inj. Avil (chlorpheniramine maleate) in 17\% was achieved, and only one case required shortcourse corticosteroids.

Conclusion: Despite $3.1 \%$ of serum sickness cases reported in our present study, the safety of ERIG has been proved and serum sickness can be managed with oral analgesics and antihistamines. Hence ERIG should be encouraged among doctors for use in category-III animal bite patients to prevent rabies.

Keywords: Equine rabies immunoglobulin, Serum sickness, Rabies
\end{abstract}

\section{Introduction}

Rabies is a major public health issue worldwide with high mortality rate. Globally, rabies is the tenth leading cause of death due to infection in humans. ${ }^{1}$ India has been quoted as reporting 30,000 human rabies deaths, accounting for nearly $60 \%$ of global mortality. ${ }^{2}$ Rabies immunoglobulin is the mainstay of treatment for severe animal bite cases. ${ }^{3}$ Equine rabies immunoglobulin (ERIG) is economical as compared to human rabies immunoglobulin (HRIG) and hence more affordable to patients in developing countries, like India. In spite of high purification techniques adopted

Corresponding Author: Dr. Tapas Ranjan Behera, Department of Community Medicine, SCB Medical College, Cuttack, Odisha, India.

E-mail Id: tapas4behera@gmail.com

Orcid Id: https://orcid.org/0000-0003-3934-6546

How to cite this article: Maharana S, Behera TR, Pattanaik N. Serum Sickness in Patients Receiving Equine Rabies Immunoglobulin. J Commun Dis 2018; 50(2): 30-33. 
for preparing ERIG, some amount of animal protein remains which causes reactogenicity, creating panic of fatal adverse effects among health care providers. Lack of knowledge on safety, fear of adverse effects mostly anaphylaxis lead to avoidance of advising and administering ERIG. ${ }^{4}$ According to the APCRI-WHO survey in India, the use of RIG is only $2 \%{ }^{2}$

Serum sickness is one of the serious side effects of ERIG. Serum sickness is a type-III or immune-complex-mediated hypersensitivity reaction that results from the injection of heterologous or foreign protein or serum. It was first described by Clemens von Pirquet and Bela Schick in 1905. ${ }^{5}$ Serum sickness after ERIG has a delayed onset between 7 and 14 days after administration. Clinically, this manifests with rashes, fever, arthralgia, myalgia, headache, and gastrointestinal symptoms. ${ }^{6}$ The safety of ERIG depends on less of side effects and also the compliance of patients. There are many studies carried out in India and abroad about the safety related to ERIG as a whole. ${ }^{3,4,7,8}$ Very few studies are there regarding serum sickness in particular. Hence this study was specifically carried out with objectives to assess the incidence, to see the clinical features, and management of cases of serum sickness following administration of ERIG.

\section{Materials and Methods}

It is a hospital-based cross-sectional study carried out between September 2016 and December 2016 at Anti Rabies Clinic (ARC) of SCB Medical College and Hospital, Cuttack, Odisha. ERIG is available free of cost for category-III animal bites at the hospital. On an average, 40-50 patients with category-III animal bites are being administered ERIG per day. Many patients are also referred from different districts to this ARC for ERIG administration as SCB Medical College \& Hospital acts as a referral hospital. Out of 3041 animal bite patients reported during the study period, 1548 (50.9\%) category-III patients received ERIG and thus were included in the study. Those cases unwilling to take ERIG and a few patients who had taken HRIG were excluded from the study. A predesigned, pre-tested, semi-structured questionnaire was used to collect data from the cases.

Biologicals Used: ERIG - Rabies Anti Serum (B. No. O2AR15007) with a potency of $300 \mathrm{IU} / \mathrm{mL}$, manufactured by ViNS Bioproducts Limited, Hyderabad, India.

Follow Up: 1548 patients were followed up for adherence to treatment and observed for any adverse effect on their next days of visit for ARV, i.e., on day 3, 7, and 28.
Data collected was entered into MS Excel and then analyzed using SPSS 21 version software. Categorical data was represented using percentages and proportions. To test the association, Chi-square test was used for categorical variable and $\mathrm{P}<0.05$ was considered statistically significant.

\section{Results}

Among all animal bite cases of category-III attending the ARC during the study period $(n=1548)$, dog bite cases are most common (74.72\%). Out of total 1548 category-III exposure patients, who received ERIG, only 48 cases (3.1\%) showed serum sickness. Most cases were between 15 and 60 years of age $(62.5 \%)$ and males (57\%). Mean age was $37.69 \pm 18.82$ years.

Among all the serum sickness cases $(n=48)$, the site of bite was most commonly found over lower limbs, i.e., legs, thigh, feet; hence site of administrating ERIG is most common in lower limbs. $66.7 \%$ of serum sickness patients had weight in the range of $60-80 \mathrm{~kg}$ (Table 1). Mean weight was $63.34 \pm 19.85 \mathrm{~kg}$.

Serum sickness manifested in form of rashes in $97.82 \%$ cases, fever in $85.41 \%$, itching in $77.08 \%$, malaise in $58.34 \%$, other symptoms like angioedema, edema, and gastrointestinal symptoms in $6.25 \%$ only (Table 2 ).

All the patients $(n=48)$ developed signs and symptoms of serum sickness after seven days of ERIG administration, Majority of them (48\%) presented with signs of serum sickness on eighth day. Similarly, $29 \%$ presented on ninth day, $12 \%$ presented on tenth day and $11 \%$ reported on eleventh day. However, no case of serum sickness was reported after 14 days of ERIG administration.

Different simple modalities of treatment were given to these patients $(n=48)$ based on the acuteness and severity of the signs and symptoms for symptomatic relief with oral antihistamines like Cetirizine and Levocetirizine in $60 \%$ of cases, Inj. Avil (chlorpheniramine maleate) in $17 \%$ of cases and only one case required corticosteroids. However, $21 \%$ of cases required simple analgesic (Aceclofenac and Paracetamol) for symptoms like fever and malaise. All the signs and symptoms in these 48 patients subsided within 10 days of treatment.

Day of disappearance of sign and symptoms following treatment were within 3 days in $29 \%$ cases, within 3-5 days in $39.2 \%$ of cases and within $5-10$ days in $31.8 \%$ of cases. 
Table 1.Demographe Profile of Patients Receiving ERIG at ARC (N=1548)

\begin{tabular}{|c|c|c|c|}
\hline Patient Profile & $\begin{array}{l}\text { No. of Serum Sickness } \\
\text { Cases }(\mathrm{N}=48)\end{array}$ & $\begin{array}{l}\text { No. of Patients not Developed Serum } \\
\text { Sickness }(\mathrm{N}=1500)\end{array}$ & P-value \\
\hline \multicolumn{4}{|l|}{ Age } \\
\hline$\leq 5$ years & $2(4.1 \%)$ & $57(3.8 \%)$ & \\
\hline $5-14$ years & $4(8.3 \%)$ & $422(28.1 \%)$ & 0.005 (significant) \\
\hline $15-60$ years & $30(62.5 \%)$ & $836(55.7 \%)$ & \\
\hline$>60$ years & $12(25 \%)$ & $185(12.3 \%)$ & \\
\hline \multicolumn{4}{|l|}{ Sex } \\
\hline Male & $25(52.08 \%)$ & $876(58.4 \%)$ & 0.38 \\
\hline Female & $23(47.9 \%)$ & $624(41.6 \%)$ & \\
\hline \multicolumn{4}{|l|}{ Type of Animal Bite } \\
\hline Dog & $35(72.92 \%)$ & $916(61 \%)$ & 0.28 \\
\hline Cat & $7(14.58 \%)$ & $337(22.4 \%)$ & \\
\hline Monkey & $5(10.41 \%)$ & $150(10 \%)$ & \\
\hline $\begin{array}{c}\text { Others } \\
\text { (beer, jackal, fox, etc.) }\end{array}$ & $1(2.08 \%)$ & $97(6.4 \%)$ & \\
\hline \multicolumn{4}{|l|}{ Site of Animal Bite } \\
\hline Upper limb & $10(20.8 \%)$ & $548(36.5 \%)$ & \\
\hline Lower limb & $28(58.33 \%$ & $563(37.5 \%)$ & \\
\hline Trunk & $2(4.16 \%)$ & $142(9.4 \%)$ & 0.03(significant) \\
\hline Head and neck & $2(4.16 \%)$ & $85(5.6 \%)$ & \\
\hline Multiple sites & $6(12.54 \%)$ & $162(10.8 \%)$ & \\
\hline \multicolumn{4}{|l|}{ Weight (in kg) } \\
\hline$<20$ & $4(8.3 \%)$ & $68(4.5 \%)$ & \\
\hline $20-40$ & $4(8.3 \%)$ & $223(14.8 \%)$ & 0.001 (significant) \\
\hline $40-60$ & $8(16.6 \%)$ & $694(46.2 \%)$ & \\
\hline $60-80$ & $32(66.7 \%)$ & $515(34.3 \%)$ & \\
\hline
\end{tabular}

Table 2: Distribution of Different Signs and Symptoms of Serum-Sickness-Like Reactions Presented by 48 of the 1548 Patients Who Received Equine Rabies Immune Globulin (ERIG) and Were

Followed Up for at Least 1 Month

\begin{tabular}{|c|c|}
\hline Sign and Symptoms & No. of Cases \\
\hline Rashes & $47(97.82 \%)$ \\
\hline Fever & $41(85.41 \%)$ \\
\hline Pruritus & $37(77.08 \%)$ \\
\hline Malaise & $28(58.34 \%)$ \\
\hline Others (angioedema, edema, gastrointestinal symptoms) & $3(6.25 \%)$ \\
\hline
\end{tabular}

\section{Discussion}

Most of the patients enrolled in this study had been bitten by dogs, the main reservoir for rabies in India. Currently in developing countries, less than $2 \%$ of all post-exposure treatment includes both vaccine and serum. This is because HRIG is not widely available and its cost is prohibitive and there is apprehension about the side effects of ERIG, especially serum sickness. Purification techniques today have reduced the risk of sensitization to ERIG. In our study, the incidence of serum sickness among recipients was reported to be 3.1\%. A study by Wilde et al. at the Queen Saovabha Memorial Institute, Bangkok, among 3156 patients who were treated with ERIG found that only 51 patients (1.6\%) exhibited serum-sickness-like reactions. ${ }^{3}$ In the present study, almost equal number from both sexes exhibited serum-sickness-like reactions (52.08\% of males) whereas Wilde et al. in their study found the incidence more among females. In the present study, more patients were in the age group of 15-60 years. Wilde et al. in their study mentioned the incidence among children rarely exceeds $0.086 \%$, but in our study incidence among children (age group $0-14$ years) is $0.125 \%$. Most of the patients had weight in the range of $60-80 \mathrm{~kg}$, i.e., most cases received large amount of ERIG. Wilde et al.'s study also showed few cases of lymphadenopathy while in our study there is no case of lymphadenopathy. ${ }^{3}$ Behera et al. in a study at MKCG Medical College, Berhampur, Odisha, India, found incidence of serum sickness to be $3 \% .{ }^{4}$ Similarly, in a study by Behera et al., among 195 patients who were injected ERIG over 
fingers and toes, reported $1.53 \%$ of delayed serum sickness reactions at MKCG Medical College, Berhampur, Odisha. ${ }^{9}$ Pain was the most common side effect in many studies receiving immunoglobulin. ${ }^{7}$ Another study by Wilde et al. noted adverse serum sickness like illness of 3.58\%. ${ }^{10}$ According to a previous study by Sikes in 1969, the less purified ERIG had a higher incidence of serum sickness of $16 \% .^{11}$

In the present study, majority of the patients (48\%) presented with signs of serum-sickness-like reaction on eighth day and none reported after 14 days of ERIG administration which coincides (after seven days of ERIG administration) with a previous study by the author at MKCG Medical College, Berhampur, Odisha. ${ }^{9}$ Similar finding was also reported by Wilde et al. where they found the cumulative days of appearance of serum-sickness-like reaction from 1-15 days after ERIG administration with highest peak of occurrence from 7-15 days post ERIG administration. ${ }^{3}$

In our study, the serum-sickness-like reactions were slightly more marked in males than females (52.08\% vs $47.92 \%$ ) and only $2 \%$ cases were treated with corticosteroids. However Wilde et al. in their study found serum-sickness-like illness more frequent among females and 15\% were treated with short course of corticosteroids. Similar to our study, none of serum-sickness-like illness persisted for more than a week as reported by Wilde. ${ }^{3}$ According to a previous study by Sikes in 1969 , this incidence increases to $46 \%$ for persons over 15 years of age. ${ }^{11}$ Recently, due to high purification techniques ERIG shows very low incidence of serum sickness.

\section{Conclusion}

This prospective study of 1548 patients with category-III animal bites treated with ERIG suggested that despite occurrence of serum sickness in $3.1 \%$ of cases, the administration of ERIG is a safe and lifesaving procedure.

The signs and symptoms of serum sickness can be managed at the outpatient department of the anti-rabies clinic with oral antihistamines and analgesics.

Therefore, it is concluded that careful follow up of the patients with category-III animal bites treated with ERIG is required to complete the treatment despite only a few occurrences of serum-sickness-like symptoms for prevention of rabies.

\section{Conflict of Interest: None}

\section{References}

1. WHO drug information, World Health Organization, Geneva, Switzerland. 2002; 16(1): 4-5.

2. Sudarshan MK, Madhusudana SN, Mahendra BJ et al. Assessing the burden of human rabies in India: Results of a national multi-center epidemiological survey. International Journal of Infectious Diseases 2007; 11: 29-35.

3. Wilde $\mathrm{H}$, Chomchey $\mathrm{P}$, Punyaratabandhu P. Purified equine rabies immune globulin: A safe and affordable alternative to human rabies immunoglobulin. Bulletin of the WHO1989; 67(6): 731-36.

4. Behera TR, Satapathy DM, Pratap AK. Post-exposure prophylaxis for rabies with ERIG and IDRV in children. J Commun Dis 2011; 43(1): 31-37.

5. Nicole MR, Renai TK, Simon GA et al Incidence of serum sickness after the administration of Australian snake antivenom (ASP-22). Clinical Toxicology 2016; 54(1): 27-33.

6. Yerushalmi J, Zvulunov A, Halevy S. Serum sickness-like reactions. Cutis 2002; 69(5): 395-97.

7. Satpathy DM, Sahu T, Behera TR. Equine rabies immunoglobulin: A study on its clinical safety. J Indian Med Assoc 2005 Apr; 103(4): 238, 241-42.

8. Salva EP, Dimaano EM, Villarama JBR et al. An evaluation of the and potency of equine rabies immunoglobulin through safety measurement of suppression on vaccine-induced antibody production among healthy volunteers. Philippine Journal of Internal Medicine 2014; 52(2): 1-7.

9. Behera TR, Satapathy DM, Pratap AK. Safety of equine rabies immunoglobulin injection into finger and toes. Asian Biomedicine Jun 2012; 6(3): 429-32.

10. Wilde $\mathrm{H}$, Chutivongse $\mathrm{S}$. Equine rabies immune globulin: A product with an undeserved poor reputation. Am J Trop Med Hyg 1990; 42(2): 175-78.

11. Sikes RK. Human rabies immune globulin. Pub Health Report Sep 1969; 84(9): 797-800.

Date of Submission: 2018-04-17 Date of Acceptance: 2018-05-01 